\title{
L'administration locale en temps de crise : le cas de l'Isère en 1814-1815
}

\section{Marie-Cécile Thoral}

\section{(2) OpenEdition \\ 1 Journals}

\section{Édition électronique}

URL : https://journals.openedition.org/ahrf/2144

DOI : 10.4000/ahrf.2144

ISSN : 1952-403X

Éditeur :

Armand Colin, Société des études robespierristes

\section{Édition imprimée}

Date de publication : 1 mars 2005

Pagination : 117-135

ISSN : 0003-4436

\section{Référence électronique}

Marie-Cécile Thoral, "L'administration locale en temps de crise : le cas de l'Isère en 1814-1815 »,

Annales historiques de la Révolution française [En ligne], 339 | janvier-mars 2005, mis en ligne le 15 mars 2008, consulté le 23 avril 2022. URL : http://journals.openedition.org/ahrf/2144 ; DOI : https://doi.org/ 10.4000/ahrf.2144

Ce document a été généré automatiquement le 23 avril 2022.

Tous droits réservés 


\title{
L'administration locale en temps de crise : le cas de l'Isère en 1814-1815
}

\author{
Marie-Cécile Thoral
}

1 Par sa loi du 28 pluviôse an VIII (17 février 1800), Napoléon met en place une nouvelle organisation administrative. Celle-ci est soumise à rude épreuve lors de la chute de l'Empire et des guerres qui l'accompagnent. À partir du printemps 1813, et jusqu'à l'abdication de Napoléon en avril 1814 (et à nouveau à la fin des Cent Jours, au printemps 1815), les faits militaires dominent la vie politique française. Les événements se précipitent à partir de l'été 1813 , après la formation de la coalition des Alliés, regroupant tous les adversaires de Napoléon. L'Empire napoléonien se désintègre en partie dans l'automne 1813. Au début de 1814, la France est encerclée et directement menacée sur ses frontières, ce qui n'avait plus été le cas depuis la Révolution. Entre la France du Nord et du Nord Est, le Sud-Ouest aquitain et le Sud-Est, les Alliés envahissent et occupent plus d'un tiers du sol français dans le printemps 1814 . Pourtant, à part un colloque organisé en 1989 par Yves-Marie Bercé ${ }^{1}$, et qui se concentre uniquement sur l'année 1814 et en envisageant essentiellement la situation des États annexés et la dislocation de l'Empire napoléonien en Europe, aucune étude n'a été consacrée spécifiquement à l'administration locale en ces deux années troublées.

2 Les années 1814-1815 voient se succéder, en Isère, deux guerres sur le sol du département et deux périodes d'occupation militaire ${ }^{2}$. Le sujet de cet article est le fonctionnement, pendant ces années troublées, de l'administration locale civile (administration préfectorale, administrations municipales, et quelques autres administrations comme celles des finances, les douanes ...), à l'exclusion de l'administration militaire ou de l'administration religieuse. Les fonctionnaires (agents nommés et rétribués par l'État ou par l'un de ses organes déconcentrés comme les municipalités) et les administrateurs locaux (personnes prenant part à l'administration locale, nommées par l'État, mais non rémunérées : maires, adjoints, conseillers locaux) voient leur travail, et leurs conditions de travail, évoluer sous l'effet de la guerre et de l'occupation. Ces mois de guerre et d'occupation étrangère voient une augmentation du pouvoir des militaires, ainsi qu'une désorganisation de l'administration locale civile. 
L'étude des conséquences de ces événements sur le personnel administratif, sur les tâches administratives et les conditions de travail des fonctionnaires et des administrateurs locaux permet de montrer la nécessité du recours aux citoyens, notamment, mais pas seulement, les notables locaux, pour assurer une défense du territoire efficace, protéger les intérêts de l'état et du département pendant l'occupation, et éviter une vacance du pouvoir absolue.

I. Les fonctionnaires et administrateurs locaux dans la tourmente

3 Dans l'ensemble, en Isère, les institutions administratives existantes (préfets, souspréfets, maires) sont conservées pendant cette période. Sous la première occupation (avril-mai 1814), les Alliés ont conservé les institutions à la fois par souci d'efficacité et par manque du temps nécessaire pour mettre en place d'autres institutions. Sous la seconde occupation, ils ont remplacé certaines institutions (préfet et sous-préfets) par des comités de notables choisis par eux, les «commissions administratives ", mais ce changement n'est que provisoire.

4 En revanche, à l'occasion de certains changements de régime pendant cette période, les hommes changent. L'épuration administrative de la première Restauration est très limitée en Isère, comme ailleurs en France, car ceci correspond aux vœux de Louis XVIII. Le préfet Fourier, préfet sous l'Empire, reste à la tête du département, comme beaucoup d'autres préfets impériaux ${ }^{3}$. Dans les arrondissements, les sous-préfets avaient quitté leur poste à l'approche des troupes ennemies et avaient été remplacés provisoirement par les Alliés, mais trois des quatre anciens sous-préfets sont ensuite réintégrés dans leurs fonctions par les Alliés ${ }^{4}$. Au niveau des mairies, quelques maires ont quitté leur poste à l'approche des Austro-Sardes et ne sont pas revenus par la suite, mais ils sont assez peu nombreux dans ce cas et beaucoup de maires, en Isère et dans d'autres départements comme l'Eure ${ }^{5}$, restent à leur poste, à commencer par les maires des deux plus grandes villes du département, Renauldon à Grenoble et Guillermin à Vienne, tous deux en fonction depuis le Consulat.

Le passage de la première Restauration aux Cent Jours entraîne un renouvellement plus marqué du personnel administratif. Fourier, qui s'était enfui à l'approche de Napoléon, est suspendu et remplacé par Colaud de la Salcette, conseiller de préfecture. L'Isère n'est pas un cas isolé. Seuls dix préfets en poste avant les Cent Jours gardent leurs fonctions après le retour de Napoléon. C'est le cas, par exemple, du préfet Bossi dans la Manche ${ }^{6}$. L'épuration administrative atteint aussi, en Isère comme dans beaucoup d'autres départements, le personnel préfectoral subordonné : trois des quatre souspréfets, ainsi que le secrétaire général de la préfecture. L'Isère s'inscrit ainsi dans la moyenne nationale puisque Nicolas Richardson constate que dans 66 des 87 départements, la moitié ou plus de la moitié des sous-préfets ont été remplacés ${ }^{7}$, et que $40 \%$ des secrétaires généraux en fonction à la fin de la première Restauration sont remplacés sous les Cent Jours ${ }^{8}$. Cette volonté gouvernementale de purge du personnel préfectoral s'explique par le contexte de préparation à la guerre dans lequel s'effectue l'épuration, contexte qui rend nécessaire l'installation, dans ces postes stratégiques, d'hommes sûrs, compétents et ayant la confiance publique ${ }^{9}$. L'épuration administrative atteint aussi, en Isère, une grande partie de la municipalité de Grenoble : le maire de Grenoble et deux de ses trois adjoints. Cependant, cette épuration reste malgré tout limitée, d'une part, en raison de la courte durée des Cent Jours, et, d'autre part, parce qu'un grand nombre de fonctionnaires et d'administrateurs en poste au début des Cent 
Jours ont, du fait de l'absence d'épuration sous la première Restauration, été nommés sous l'Empire ${ }^{10}$.

6 L'épuration administrative la plus forte est celle qui a lieu sous la seconde Restauration. Le préfet Bourdon de Vatry s'étant enfui, il est remplacé par une commission administrative nommée par l'intendant de l'armée d'Italie ${ }^{11}$ le 11 juillet 1815 et composée de notables locaux qui ont déjà une expérience de l'administration. Dans les arrondissements, les sous-préfets, ayant fui eux aussi, sont d'abord remplacés provisoirement, certains par des commissions administratives composées de notables locaux nommés par les Alliés, puis par des sous-préfets nommés aussi par les Alliés. Sur les quatre sous-préfets en poste avant la seconde occupation, seul un, Sapey, est conservé. À Saint Marcellin, c'est l'ancien sous-préfet qui revient à la tête de l'arrondissement: Rolland de Ravel, nommé en 1811, s'étant rallié au roi en 1814 et ayant été destitué sous les Cent Jours. Dans les deux autres arrondissements, les Alliés nomment des hommes nouveaux. Les Alliés nomment aussi un nouveau maire pour la ville de Grenoble. Avec l'approbation indispensable de l'intendant de l'armée d'Italie, la commission administrative rétablit dans leurs fonctions, le 13 juillet, tous les maires, adjoints et conseillers municipaux en fonction au 1er mars $1814^{12}$ et nomme 57 maires et adjoints nouveaux dans des villages de moins de 500 habitants ${ }^{13}$.

7 L'épuration administrative n'est pas la seule conséquence de ces événements sur l'administration locale. On constate aussi un changement dans les rapports de pouvoir entre fonctionnaires civils et militaires. Dès le début de l'année 1814, les autorités militaires voient leur influence et leur pouvoir augmenter au sein de l'administration locale, au détriment des autorités civiles. Les civils et les militaires sont amenés, en temps de paix, à se côtoyer, mais aussi à collaborer pour toutes sortes de missions locales (maintien de l'ordre, conscription...). Mais ces rapports entre civils et militaires s'intensifient en 1814 et à nouveau sous les Cent Jours, comme dans d'autres périodes de guerre comme en 1870 ou en 1914-1918 ${ }^{14}$. Lors de la défense du territoire, les autorités civiles doivent en effet collaborer avec les autorités militaires pour l'approvisionnement de l'armée et des villes, pour la formation des gardes nationales et corps francs, pour les travaux d'amélioration des fortifications, ou encore pour la mise en place d'un système de courrier plus rapide que le courrier officiel par la poste. Ce sont les militaires qui semblent avoir le véritable pouvoir, et les autorités civiles, y compris le préfet, se contentent souvent, en ce printemps de 1814, de transmettre les ordres des autorités militaires aux fonctionnaires civils et aux maires. Par exemple, le général de brigade Daumas écrit au préfet, le 3 janvier 1814 : «Il est instant que vous donniez des ordres aux maires de Voreppe, de Voiron et sur toute la route du Pont de Beauvoisin de faire une garde exacte et active pour être prévenu des mouvements de l'ennemi et pouvoir en avoir des nouvelles à chaque instant " ${ }^{15}$. Ce pouvoir croissant des militaires s'exprime et s'explique aussi par une augmentation de leurs effectifs, et surtout par une augmentation du nombre des autorités militaires locales. Des officiers d'autres départements, comme les généraux des bataillons de l'intérieur envoyés en renfort sur les frontières par exemple, viennent renforcer les effectifs de l'état-major au printemps 1814. Il y a donc, en ce début de 1814, alors que les bruits de bottes se font entendre au loin (Genève est prise en janvier 1814 par le général Autrichien Bübna, la Savoie est envahie par d'autres colonnes autrichiennes peu après), un renforcement net, et visible, non seulement des troupes (grâce notamment au rappel des militaires 
retraités) mais aussi de l'état-major, alors que le personnel des fonctionnaires civils ne bouge pas.

8 Par ailleurs, certains fonctionnaires militaires voient leur rôle fortement augmenter dans cette période de préparation à la guerre. C'est le cas notamment des commissaires ordonnateurs. Le caractère vital de leurs fonctions (assurer l'approvisionnement et le logement des troupes), surtout en temps de guerre, leur donne une place centrale à cette période et les amène à collaborer activement avec les fonctionnaires et administrateurs civils pour ordonner des réquisitions. En Isère comme en Seine-et-Oise ${ }^{16}$ et vraisemblablement comme dans tous les départements exposés à l'invasion, préfets, sous-préfets et maires ont donc des rapports réguliers, et parfois conflictuels, avec ces commissaires ordonnateurs.

De nouveaux fonctionnaires civils font aussi leur entrée sur la scène locale à cette époque : les commissaires extraordinaires, chargés des pleins pouvoirs en matière de levée des troupes, d'approvisionnement et de police politique, envoyés par le gouvernement dans les divisions militaires à partir du 2 janvier 1814. Au printemps 1815, Napoléon remet en activité le système des commissaires extraordinaires et envoie un ancien militaire, Bourdon de Vatry, dans la 7e division. Il est d'ailleurs intéressant de noter que lorsque, début mai 1815, Napoléon exige d'avoir pour l'Isère un «préfet actif » pour accélérer la défense du territoire, il nomme à ce poste cet ancien militaire, Bourdon de Vatry ${ }^{17}$. La nomination d'un ancien militaire comme préfet dans une période politique et militaire troublée et au cours de laquelle les rapports entre fonctionnaires civils et militaires sont plus fréquents a en effet de nombreux avantages. Vincent Cuvilliers a ainsi souligné que, après la reprise de la guerre contre l'Angleterre et l'augmentation du nombre de militaires dans le Pas-de-Calais, le passé militaire du préfet du Pas de Calais nommé en l'an XI, de la Chaise, «devient un atout pour faire face aux divers problèmes nés de la cohabitation entre l'armée et les autorités civiles $[\ldots] »^{18}$.

10 L'importance des militaires dans l'administration locale augmente encore après que les places militaires de l'Isère (Grenoble et fort Barraux) aient été officiellement déclarées en état de siège, le 1er mai 1815. La loi du 10 juillet 1791 et le décret impérial du 24 décembre 1811 donnent alors aux autorités militaires «tous les pouvoirs qui appartenaient précédemment aux officiers civils et ceux-ci ne peuvent plus exercer que l'autorité qui leur est déléguée par le commandant ${ }^{19}$. Ainsi toutes les attributions du maire de Grenoble sont transférées dans les mains du maréchal de camp. Cette redistribution des cartes ne se fait pas sans heurts. Les accrochages entre autorités civiles et militaires au cours de ces mois de défense du territoire témoignent de la difficulté, pour les civils, de s'effacer devant les militaires. Un bon exemple de cela est fourni par les résistances apportées par le maire à l'œuvre de fortification de la place de Grenoble. En effet, à Grenoble et fort Barraux, comme dans toutes les places de l'est de la France, comme Metz par exemple ${ }^{20}$, les Cent Jours sont une période de travaux de fortification intenses, afin de renforcer le dispositif de défense. Les soldats du génie et d'autres armes, aidés par des habitants réquisitionnés, s'occupent de fortifier, améliorer, blinder les installations militaires. Le commandant du génie demande alors aux propriétaires de maisons situées près des remparts de la ville de Grenoble d'abandonner une partie de leur terrain si celui-ci gêne la construction des murailles. Le maire de Grenoble refuse, en juin 1815, de se soumettre à la réquisition faite par le commandant du génie et par le maréchal de camp, commandant supérieur de la ville en 
état de siège, de faire démolir sans délai le mur de clôture de sa propriété située près du rempart de la ville. Le maréchal de camp écrit au maire, le 24 juin : "Je m'attendais, d'après la conférence que j'eus avec vous il y a huit ou dix jours, que vous donneriez l'exemple de soumission aux lois et ordres supérieurs, mais loin de là vous vous êtes borné à mettre un ouvrier seulement qui a enlevé 50 à 60 pierres, et cette démolition qui devait être faite dans deux ou trois jours durera plus de six mois par le peu de bonne volonté que vous y apportez [...] ${ }^{21}$, et il le menace de provoquer des mesures de rigueur. Les conflits entre le maire et le maréchal de camp prennent une telle ampleur que le lieutenant général porte l'affaire devant le Ministre de l'Intérieur. En effet, le maire de Grenoble ne se contente pas d'exprimer discrètement son mécontentement: il multiplie les provocations, comme des déclarations publiques sur le fait que, selon lui, les autorités militaires administrent mal et préparent mal le département à la guerre. Le commissaire chargé par intérim du portefeuille de l'Intérieur écrit ainsi au préfet, le 26 juin 1815 : «Je suis instruit [...] que le maire de Grenoble a fait afficher des avis dans lesquels il donne à entendre que les dispositions prises par l'autorité militaire pour la défense de la place l'ont été contre son gré. De pareilles publications sont illégales et dangereuses. Le maire n'a pas le droit de proclamer son jugement sur des mesures qui ne sont pas de sa compétence [...] Le maire ne peut sans excéder ses pouvoirs et sans compromettre gravement sa responsabilité publier des avis qui tendraient directement ou indirectement à empêcher ou à retarder l'exécution des ordres émanés de l'autorité militaire. Il devrait sentir que la conséquence d'un semblable procédé serait de montrer aux citoyens une sorte de lutte entre les agents du gouvernement, de faire naître, sinon de la résistance, du moins de l'hésitation, d'entraver, dans ces circonstances critiques, les opérations qui ont pour but la défense du territoire" ${ }^{22}$. Il est vrai que la multiplicité des conflits entre autorités civiles et militaires au début du XIXe siècle, en temps de paix, pour des questions de juridiction (notamment autour de la question du maintien de l'ordre) ou même pour de simples questions de préséances, pouvait laisser entrevoir les difficultés entraînées par la suprématie des militaires dans l'administration locale en période de préparation à la guerre et de guerre.

II. Administrer un département en temps de crise

11 Les événements de ces deux années ont aussi des conséquences sur l'activité administrative elle-même.

12 L'impératif premier consiste à éviter une vacance administrative. Pour cela, les consignes données aux fonctionnaires et administrateurs sont de rester à leur poste et de continuer leurs fonctions aussi longtemps que possible. Dans l'ensemble, la consigne est suivie au début de 1814. Plusieurs maires écrivent au préfet pour lui indiquer qu'en dépit du danger qui se rapproche, ils continuent de diriger leur commune. Lorsque les ennemis sont aux portes de la commune, les fonctionnaires ont pour ordre de s'enfuir, car un fonctionnaire restant dans une commune occupée par l'ennemi est considéré comme coupable de trahison. Mais l'évacuation de la commune ne signifie pas pour autant l'arrêt des fonctions administratives. En effet, en cas d'invasion ennemie, les fonctionnaires doivent se replier à l'intérieur, dans la zone non occupée, et y poursuivre leur travail. Ainsi, les sous-préfets doivent, au cas où le chef-lieu d'arrondissement dans lequel ils résident serait envahi, fuir la sous-préfecture et rejoindre l'armée en campagne afin de collaborer avec les officiers en leur faisant part de leur connaissance des localités et des hommes. Les fonctionnaires doivent parfois poursuivre leur travail dans des conditions qui paraissent assez surprenantes. Par 
exemple, les douaniers doivent, en théorie du moins, continuer leurs opérations (vérification des passeports, contrôle des marchandises...) comme si de rien n'était, sur une frontière mouvante, correspondant en fait à la ligne de front. Jean Clinquart a montré le caractère irréaliste et inapplicable de cette "politique des lignes mobiles » ${ }^{23}$. Les consignes du directeur de l'administration des douanes du département à ses employés, en décembre 1813, sont pourtant claires : «En cas d'invasion [...] la ligne des douanes ne doit jamais être rompue mais [...] il faut se replier dans l'intérieur à mesure que l'ennemi avance, de façon que cette ligne s'étend aujourd'hui depuis le département du Jura jusqu'au Pont de Beauvoisin. Et comme leur devoir est de s'opposer constamment à l'introduction des marchandises prohibées ou d'assurer les droits de douane, il leur est expressément recommandé de ne point agir militairement et de résister à tous les ordres des généraux à cet égard ${ }^{24}$. Ceci n'est pas une spécificité de l'Isère, mais correspond à la volonté de Napoléon d'empêcher que les douaniers ne soient détournés de leurs fonctions ${ }^{25}$, et notamment de la lutte contre la contrebande.

Cependant, cette volonté de continuer à administrer le département coûte que coûte comme en temps de paix se heurte à deux obstacles: l'attitude des administrés et la menace d'invasion.

14 L'administration civile est désorganisée par la réaction de la population locale face à la menace extérieure, la guerre et l'occupation. La peur engendrée par l'approche des armées ennemies crée un climat de panique et de quasi-anarchie dans lequel les devoirs civiques semblent oubliés de la part des administrés. Ainsi, les employés des contributions indirectes constatent, dans la majorité des communes, au début de 1814, un refus manifeste de la part des habitants de payer leurs contributions. Ceci peut aussi s'expliquer par l'augmentation de la fiscalité depuis 1813. Néanmoins, la raison principale de cette résistance semble être une méfiance, ou même une hostilité croissante envers les représentants de l'État. Le préfet reçoit ainsi, au printemps 1814, une lettre anonyme dans laquelle il est écrit : «Des projets insensés se forment contre la sûreté des agents du gouvernement que le public dans son extrême agitation accuse de trahison $[. .]. »{ }^{26}$. Le contrôleur principal des Droits Réunis de Vienne se plaint en effet de l'insécurité grandissante due en grande partie à l'hostilité marquée des habitants : «Ce matin, à cinq heures, Monsieur le contrôleur de ville et Monsieur le receveur principal étaient partis. Messieurs les employés paraissent s'occuper de leur sûreté personnelle; je reste seul avec Messieurs les contrôleurs de l'octroi. Le service est totalement désorganisé. Hier les cabaretiers et autres assujettis ont menacé les employés qui ont été obligés de se retirer pour éviter les voies de fait [...] Je ne sais si je pourrai tenir longtemps à mon poste. La populace devient insolente et je ne puis sortir sans entendre des propos désagréables $"{ }^{27}$. Cette hostilité était latente sous le Consulat et sous l'Empire, notamment dans les campagnes, où les fonctionnaires, et notamment les gendarmes et les fonctionnaires des finances, étaient très mal perçus par la population, mais les circonstances de crise, et surtout le mécontentement de la population envers les autorités locales accusées de laisser le département sans défense efficace, mettent le feu aux poudres et entraînent une résistance généralisée envers les fonctionnaires sur le terrain, faisant un peu figure de boucs émissaires. Cette hostilité massive de la population locale à l'égard des fonctionnaires pourrait entraîner de fait, comme le constate Paul Janssens pour les départements belges en 1814, une vacance du pouvoir car «la vacance du pouvoir ne dépend pas seulement de la présence ou de l'absence des autorités civiles. Encore faut-il que les fonctionnaires en place se fassent 
obéir de la population. Si leur pouvoir est contesté, une vacance de fait du pouvoir risque de s'installer [...] " ${ }^{28}$. Paul Janssens évoque ainsi, pour les départements belges, une « décomposition de l'autorité française, sous le coup des défaites successives et de la menace imminente que constitue l'approche des alliés pour le pouvoir en place [...] » ${ }^{29}$ : refus de la population locale de payer les contributions, désertion de la conscription, " désobéissance civile généralisée », émeutes ${ }^{30}$...

La tâche des fonctionnaires des finances, face à cette résistance massive, devient pratiquement impossible à accomplir en Isère en janvier 1814, car ils doivent faire face à des insultes, des menaces et même des coups ${ }^{31}$.

Par ailleurs, la menace d'invasion empêche aussi souvent le fonctionnement normal des différents services. Avec la préparation de la guerre et la défense du territoire, de nouvelles missions apparaissent pour les fonctionnaires et administrateurs locaux : la formation de gardes nationales actives ou de corps francs, le rappel des militaires retraités, l'organisation de l'approvisionnement, l'envoi d'éclaireurs pour se tenir informés des mouvements de l'ennemi. D'autres missions, comme la traque des conscrits réfractaires ou la surveillance des étrangers, déjà exercées en temps de paix, revêtent une importance plus grande en cette période. Il y a une "militarisation » de l'administration départementale et municipale. Dans les bureaux de la préfecture et des sous-préfectures, les tâches militaires l'emportent sur les autres tâches. Beaucoup d'affaires sont mises en attente, les employés devant avant tout s'occuper d'opérations comme le départ des conscrits ou les réquisitions. Le préfet assure ainsi, en janvier 1814: "Tout notre temps et nos moyens sont employés jour et nuit à déférer aux demandes et réquisitions continuelles de l'autorité militaire [...] ${ }^{32}$. Quant aux maires, ils se voient investis de missions militaires comme la collecte de renseignements stratégiques, ou encore les réquisitions de bois destinées au blindage du pont-levis de fort Barraux ${ }^{33}$.

L'importance de certaines tâches et la nécessité d'agir rapidement entraînent aussi une augmentation de l'autonomie des maires à cette période. Ceci est particulièrement vrai, face à la menace d'invasion, pour les communes frontalières, les plus exposées aux dangers. Ainsi, le maire de Pont-de-Beauvoisin, commune frontalière avec la Savoie, informe le préfet, le 5 janvier 1814, qu'il a pris, de sa propre autorité, une mesure consistant à enchaîner sur la rive gauche du Rhône les trois bacs et bateaux stationnés sur ce fleuve depuis la Balme (en Savoie) jusqu'à Saint-Didier, afin que l'ennemi ne puisse les utiliser pour traverser le fleuve. Le maire avoue: «J'ai outrepassé mes pouvoirs en prenant cette mesure provisoire de précaution et de sûreté générale ", mais il ajoute : «Elle a déjà reçu l'approbation de Monsieur le sous-préfet de la Tour du Pin et j'espère qu'elle recevra la vôtre ${ }^{34}$.

18 Sous les deux occupations, en-dehors de Grenoble où sont formés des comités de subsistances composés de notables locaux, les maires et adjoints sont souvent seuls, avec leurs administrés, face aux Alliés pour assurer l'approvisionnement de ces derniers. Jacques-Olivier Boudon note, en ce qui concerne la crise touchant le corps préfectoral en 1814-1815: « Aux traditionnelles charges dévolues aux préfets s'ajoute la relation avec les forces d'occupation [...] ${ }^{35}$, or cette relation n'est pas toujours facile. Le maire de Malbenc raconte ainsi le séjour des Alliés dans son village en 1814 : "Les troupes coalisées ont occupé la commune de Malbenc pendant près d'un mois, elles étaient au nombre de 500 dont partie hussards [...]. J'étais presque seul pour répondre à des hommes que je n'entendais presque pas, leur fournir ce qu'ils demandaient [...] je 
me hâtai de faire des réquisitions, m'estimant très heureux ainsi que les habitants si l'on parvenait à détourner la baïonnette levée sur nos têtes [...] " ${ }^{36}$. La position des maires et adjoints est d'autant plus difficile que les officiers étrangers, ne prêtant aucune attention à l'état de détresse des communes, exigent des rations alimentaires énormes pour leurs soldats, et composées uniquement de produits de luxe (pain blanc, légumes frais, viande, vin) et organisent des soirées fastueuses dans leurs quartiers généraux, financées par les populations locales. L'adjoint au maire de Voiron, troisième ville du département et siège du quartier-général autrichien en avril 1814, rapporte ainsi que les habitants durent loger et nourrir tous les soldats et "tout l'état-major auquel il fallait une table splendide et tous les objets de sensualité ; l'administration fit à cet effet une réquisition en argent montant à 8000 francs sans laquelle on n'aurait pu se procurer ces objets de luxe ${ }^{37}$.

L'exercice normal des fonctions administratives, comme en temps de paix, est aussi compromis par le fait que, face à la menace d'invasion, les fonctionnaires sont parfois obligés d'abandonner leurs fonctions pour prendre une part active à la défense du territoire. Ainsi, lorsque, au début de 1814, les Autrichiens pénètrent en Savoie et se rapprochent du département, tous les fonctionnaires locaux dont les fonctions sont voisines du maintien de l'ordre sont appelés à rejoindre les militaires au combat. Le lieutenant général donne ainsi l'ordre, le 7 février 1814, aux gardes forestiers du département d'organiser en compagnies les gardes champêtres et forestiers en état de combattre et d'y adjoindre les gardes nationaux les plus « à même de tirer un coup de fusil ${ }^{38}$. Douaniers, en Isère comme dans d'autres départements menacés de l'invasion ${ }^{39}$, gardes champêtres et gardes forestiers joignent leurs forces aux soldats de la ligne et aux gardes nationaux, partent sur le front avec les militaires et dégarnissent ainsi l'arrière de fonctionnaires civils.

Enfin, l'insécurité grandissante due à l'approche de l'ennemi force les fonctionnaires à interrompre leurs fonctions avant le déclenchement de la guerre. Des procédés d'évacuation sont mis en place. Les fonctionnaires ont en effet pour devoir de mettre en lieu sûr l'argent et les documents (registres, cartes, rôles de contributions...) de leurs administrations, ainsi que tout ce qui pourrait être utilisé par les occupants (les caisses d'argent de l'administration des Mines, les chevaux du dépôt d'étalons de Grenoble, le tabac de l'entreposeur principal des tabacs du département...). Les fonctionnaires civils s'occupent aussi, exceptionnellement, de l'évacuation des administrations militaires, ce qui est une lourde opération car l'arsenal de Grenoble sert alors d'entrepôt pour tout l'approvisionnement militaire de la frontière sud-est et renferme 200 pièces de canon et 80000 fusils, Grenoble paraissant alors la place la plus sûre, protégée par les montagnes et à l'abri de ses fortifications ${ }^{40}$. Tous ces documents et biens sont emballés et chargés sur des voitures ou, pour les produits lourds comme le tabac, sur des bateaux, afin d'être envoyés dans les départements de l'intérieur de la France, hors de portée de l'occupant. Ceci se fait souvent dans la précipitation, voire dans la panique, comme le montre le témoignage du contrôleur des Droits Réunis de Vienne, le 14 janvier 1814: "La ville est toute en mouvement, les négociants emballent leurs marchandises, les moyens de transport sont à des prix excessifs. Il est impossible de se procurer des hommes de peine pour emballer les registres et pièces de comptabilité. Ils préfèrent travailler pour les négociants. J'ai eu le bonheur de me procurer une barque pour l'évacuation des tabacs de l'entreposeur particulier ; Monsieur le procureur fondé de l'entreposeur et moi y avons travaillé toute la nuit, je les ai dirigés [...] vers l'entrepôt principal de Valence. Monsieur le procureur fondé les accompagne. Je suis 
obligé moi-même d'emballer mes états et pièces de comptabilité, j'en mettrai en sûreté le plus que je pourrai ${ }^{41}$. Le but n'est pas seulement de protéger ces documents, mais aussi de compliquer l'administration du territoire par les occupants en faisant en sorte, par exemple, que, en l'absence de rôles de contributions, il soit très difficile à l'occupant de lever des impôts. Le sous-préfet de Vienne écrit ainsi aux maires de son arrondissement, le 19 janvier 1814 : «Vous enjoindrez, au moment où l'envahissement serait inévitable, aux ministres du culte, aux percepteurs, préposés des droits réunis et de tous les deniers publics, agents de tous services, gardes champêtres et forestiers de quitter la commune après avoir pris à l'avance des mesures certaines pour la mise en sûreté de l'argent et des papiers, titres, documents de tous genres. Il est de la plus haute importance de ne rien laisser à l'ennemi de ce qui pourrait lui faciliter les moyens d'administration et surtout des levées de contributions $"{ }^{42}$.

III. L'aide nécessaire des citoyens

21 En ces temps troublés, les fonctionnaires et administrateurs doivent avoir recours, encore plus qu'en d'autres périodes, à l'aide des citoyens. Cette nécessité pratique rejoint une demande des habitants eux-mêmes. Les Dauphinois, plus par attachement à leur pays et pour défendre leurs foyers et leurs familles que par bonapartisme semble$\mathrm{t}$-il, font preuve d'un fort patriotisme. Ils se distinguent en cela de leurs voisins suisses et savoyards, qui ont accueilli les Alliés avec plaisir car la guerre avait contribué à renforcer leur sentiment national et leur volonté d'indépendance vis-à-vis de la France. Le patriotisme des habitants s'exprime aussi bien dans le grand nombre d'engagements volontaires dans les gardes nationales ou corps francs que dans l'aide matérielle et financière apportée aux soldats par les habitants. Les exemples de maires voyant affluer les volontaires pour s'enrôler dans les gardes nationales ou dans les corps francs sont nombreux. Ainsi, le maire de Pont-de-Beauvoisin, nommé commissaire civil des gardes nationales, voit se présenter devant lui, le 21 janvier 1814, 250 hommes de quatre hameaux voisins, avec trois capitaines en tête. Le maire se réjouit de ce "spectacle vraiment touchant", de ces paysans venant spontanément offrir leurs services et ayant, pour cela, amené tout ce qui pouvait leur servir d'armes : fusils de chasse, sabres, et même haches ${ }^{43}$.

L'attitude des administrateurs locaux et fonctionnaires envers ce patriotisme et cette volonté de participation des citoyens à l'effort de guerre est ambiguë. La première réaction semble être une réaction de scepticisme sur l'utilité de ces engagés volontaires. Les habitants s'en rendent compte et, frustrés de ne pouvoir prendre une part plus active à la défense de leur pays, expriment violemment leur mécontentement. Le préfet reçoit ainsi une lettre anonyme, au début de 1814, dans laquelle l'auteur écrit: «Le public extrêmement inquiet et agité ne voit pas l'extrême circonspection avec laquelle vous êtes obligé d'agir [...] La plupart des services offerts par les habitants des campagnes et les anciens militaires ont été refusés ou ajournés [...] On s'échauffe, on s'indigne, on est prêt à se livrer à tous les excès si l'ennemi approche davantage [...] Ordonnez s'il le faut que le tocsin soit sonné et que tous courent aux armes [...] ${ }^{44}$. Mais l'attitude des autorités locales s'explique plus par un pragmatisme dans la mise au point de la défense du territoire que par une méfiance politique à l'encontre de la population (méfiance à l'égard d'un peuple en armes). En effet, elles doivent tenir compte du fait que beaucoup de volontaires ne sont pas armés, que les armes manquent, et qu'un garde national sans arme n'est pas très utile. Le maire de Pont-deBeauvoisin avoue ainsi que, sur les 250 hommes qui se sont présentés le 21 janvier 1814, « il n'y en avait guère qu'1/3 armés de fusils de chasse dont la plupart en mauvais 
état ». Il a donc décidé, au vu de la revue des armes disponibles, d'écrire aux maires des communes environnantes pour diminuer les demandes d'hommes et ne demander qu'un contingent modéré, composé essentiellement des anciens militaires «que l'on armera de fusils de chasse ou de calibre en bon état, avec invitation de les pourvoir de vivres pour trois jours" ${ }^{45}$. Dans d'autres départements, comme dans les HautesPyrénées ${ }^{46}$, les administrateurs locaux choisissent de laisser se former des gardes nationales dépourvues d'armement, mais ceci se révèle totalement inefficace.

Il y a aussi une autre raison à cette prudence des autorités locales face à l'enthousiasme patriotique des habitants. Les fonctionnaires et administrateurs sont sceptiques sur l'efficacité d'amateurs dans le domaine militaire et préfèrent confier la défense du territoire à des militaires ou à d'anciens militaires et éviter les manifestations spontanées de patriotisme des villageois. Ainsi, le maire de Voiron se plaint au préfet de la confusion entraînée par le zèle patriotique des habitants des villages voisins : « Le moyen employé dans plusieurs communes qui environnent la mienne de sonner le tocsin et de marcher en tumulte contre un ennemi éloigné de plus de dix lieues est une mesure absolument fausse et pernicieuse [...] parce que des hommes qui partent en foule, sans ordre, sans vivres et mal armés ne présentent qu'une défense absolument éphémère ; occasionnant des déplacements pernicieux aux intérêts des familles sous le rapport de la dépense et de la perte du temps [...] compromettent la sûreté des pays qu'ils parcourent par le défaut de discipline [...] Sonner le tocsin lorsque l'ennemi est à dix lieues, quand on est incertain sur sa marche, on voit au premier coup d'œil tout ce qu'une pareille mesure présente de désastreux et les cris des femmes et des enfants et l'abandon des travaux de l'agriculture et de toutes les autres affaires [...] ». Il propose à la place l'établissement d'une "défense régulière ", consistant dans la formation, dans chaque canton, d'une compagnie de 100 à 150 hommes, commandés et entraînés par des militaires retraités, et qui, en cas de besoin, seraient incorporées dans les régiments et payés par le gouvernement ${ }^{47}$. Cet exemple, loin d'être isolé, est assez révélateur des problèmes rencontrés par les fonctionnaires et administrateurs locaux dans l'organisation d'une « armée citoyenne » complémentaire à l'armée de ligne. En fait, il y a plus, de la part des autorités locales, une volonté de rationalisation, d'organisation, et d'efficacité dans la défense du territoire, utilisant et canalisant le patriotisme des habitants d'une manière ordonnée, qu'une mise à l'écart volontaire de la population. Cependant, après les réticences et hésitations des premiers temps, les fonctionnaires et administrateurs locaux doivent, face à l'avancée des Alliés, avoir massivement recours aux gardes nationales actives et corps francs, tant en 1814 qu'en 1815.

En-dehors de la participation militaire, les habitants apportent aussi aux fonctionnaires et administrateurs locaux une aide précieuse dans l'administration civile quotidienne. L'aide des citoyens permet ainsi parfois de suppléer au manque de fonctionnaires, soit parce que beaucoup de fonctionnaires ont rejoint les militaires sur le front, soit parce qu'ils ont, sous les deux occupations, conformément aux ordres, quitté le territoire après l'entrée des Alliés. Un bon exemple de cela peut être donné par le maintien de l'ordre. Lors de la défense du territoire, les militaires de la troupe de ligne, les gendarmes, les gardes champêtres et forestiers étant mobilisés pour la défense des frontières, les villages, dépourvus de commissaires ou d'agents de police, et les villes, laissées à un ou deux commissaires de police et à une poignée d'agents de police, risquent d'être la proie des pilleurs et des criminels, profitant d'une situation de quasiimpunité pour commettre leurs forfaits. Pour éviter cela, dans beaucoup de communes, 
les gardes nationales sédentaires, dirigées par des notables locaux, prennent en charge le maintien de l'ordre. De plus, dans beaucoup de cas, les citoyens, pas nécessairement membres des gardes nationales, s'organisent spontanément pour défendre les hommes et les propriétés, mettant au point des systèmes de rondes et de patrouilles nocturnes ${ }^{48}$.

Les notables, particulièrement sollicités en cette période troublée, sont même appelés à assurer la direction de l'administration locale civile sous la seconde occupation, en remplaçant le préfet et, dans certains cas, les sous-préfets, et en se chargeant de la mission importante de l'approvisionnement des Alliés.

Le préfet de l'Isère nommé sous les Cent Jours s'étant enfui à l'approche des troupes alliées, il est remplacé par une commission administrative départementale composée de notables locaux. Georges Clause a noté que, dans la Marne, en 1814, les Prussiens aussi avaient préféré, plutôt que d'exercer eux-mêmes seuls les fonctions administratives, "mettre en fonction des Français, honorables et désintéressés, capables de rassurer les populations, de désarmer les jusqu'au-boutistes, et d'assurer les réquisitions et les taxes qu'ils allaient réclamer [...] ${ }^{49}$. Les occupants militaires exercent un pouvoir très étendu, plus étendu que le pouvoir civil, et établissent un contrôle très strict sur les administrateurs civils français. Par exemple, la commission administrative départementale " ni toute autre autorité ne peut frapper aucun impôt ni disposer d'aucun fonds de caisse publique [...]» sans l'autorisation de l'intendant de l'armée d'Italie ${ }^{50}$. Et toutes les décisions ou arrêtés de la commission départementale doivent être approuvés par l'intendant ${ }^{51}$. Mais, malgré ces attributions restreintes, les membres de cette commission jouent un grand rôle dans la protection des habitants et des intérêts du département et de l'État. Ils alertent ainsi à plusieurs reprises le Ministre de l'Intérieur du Roi de France sur l'exploitation du département par les Alliés, comme, en juillet 1815 , la saisie et la vente, par l'intendant, d'un certain nombre de propriétés de l'État français dans le département (bois, terres, mines, établissements publics...) ${ }^{52}$. Ils s'opposent aussi à la volonté des occupants, en 1815, de surcharger le département de dépenses. Par exemple, après avoir émis des réclamations fermes auprès du général de la Tour, général de l'armée piémontaise d'occupation, au sujet du projet des occupants de réparer les fortifications de la place de Grenoble, ce qui entraînerait une dépense énorme et inutile car « dans les circonstances actuelles [...] la ville n'est menacée par aucune force extérieure " ${ }^{53}$, ils obtiennent l'arrêt de ces travaux fin juillet 1815 .

Sous la seconde occupation, une nouvelle institution, une commission des subsistances est formée le 11 juillet 1815 à Grenoble, elle aussi composée de notables locaux. Elle supervise les achats et les distributions de vivres et divers produits, et essaie d'aider les maires des communes voisines dans lesquelles des militaires étrangers sont établis en leur envoyant des secours, prélevés dans les magasins de la ville. Grenoble constitue en effet des stocks de diverses marchandises à fournir aux occupants, stocks confiés aux soins du garde-vivre de la ville, Meffre. Les distributions sont d'autant plus contraignantes qu'elles sont toujours faites sous la menace puisque, si elles ne sont pas exécutées dans leur intégralité, les occupants infligent diverses punitions à la commune, à commencer par le placement de soldats en résidence («exécutions militaires »). Ainsi, le maire de Voreppe, paniqué, écrit au comité des subsistances de Grenoble, le 15 août 1815, après avoir reçu l'ordre de préparer la nourriture et le logement pour 1600 Autrichiens qui doivent arriver deux heures plus tard, alors que 
les habitants logent déjà 4600 hommes dans leurs maisons : « Ma position et celle de la commune devient tous les moments plus critique [...] Il est impossible que nous puissions nous tirer de là si vous n'envoyez aujourd'hui même à la réception de ma lettre et sans perdre un moment au moins 3000 rations de pain et 25 quintaux de pain blanc. Manquant d'argent nous ne pouvons nous procurer ni viande ni vin [...] Venez à notre secours ou demain matin Voreppe sera déserté et abandonné à la fureur des soldats $[. ..] »^{54}$.

29 Enfin, les citoyens, et surtout les notables, contribuent, par leur aide financière, à l'effort de guerre puis, sous les deux occupations, à la protection du département face aux occupants. Ils jouent un grand rôle dans l'approvisionnement des villes et villages, et des places militaires surtout. Ainsi, après que la ville de Grenoble ait été déclarée en état de siège, le 1er mai 1815, les autorités militaires donnent l'ordre aux habitants de faire des stocks pour pouvoir tenir éventuellement un siège de six mois. Les notables de la ville viennent alors aux secours des plus pauvres en constituant, grâce aux dons des habitants aisés, un grenier d'abondance dans la ville de Grenoble ${ }^{55}$.

Sous les deux occupations, les occupants font peser un climat de crainte dans le département. La population, terrifiée par les crimes commis par les soldats (vols, incendies, viols, assassinats), par les menaces d'« exécutions militaires» ou, pire, de pillage et d'incendie de la commune, se tournent, en quête de protection, vers les notables. Ceux-ci servent souvent d'intermédiaires entre les occupants et le peuple, notamment en donnant une grande partie de leurs récoltes ou de leurs biens pour sauver le village et ses habitants.

Conclusion

31 Ces années de crise ont été des années d'épreuve pour l'administration locale et laissent des traces même après le départ des Alliés. Les fonctionnaires et administrateurs locaux, au début de la seconde Restauration, constatent que le souvenir de l'occupation qui a accompagné les tout débuts de la seconde Restauration, et notamment le poids des réquisitions, entachent la popularité du nouveau régime et contribuent à une idéalisation des années impériales. Ainsi le sous-préfet de Vienne confie au commissaire général de police, le 23 février 1816 : «Il y a [...] une nuance à observer entre [les cantons de l'arrondissement de Vienne] que Buonaparte a traversés [...] et ceux qui ont été exempts de cette calamité, entre ceux qui ont été le plus surchargés par le séjour des troupes alliées et ceux qui l'ont été le moins. Les cantons de la Verpillière, Meyzieux et Heyrieux ayant été témoins des moments de délire qu'occasionnât le passage de l'usurpateur, et ayant été successivement plus foulés par l'occupation des troupes alliées, sont aussi ceux qui exigent la surveillance la plus active en raison de l'exaltation qu'avait fait naître dans les mauvaises têtes le passage de Napoléon ainsi que l'exaspération qu'a occasionnée le séjour des troupes alliées. C'est dans ces trois cantons qu'on a eu le plus de peine à réprimer les cris séditieux et à faire acquitter les impôts $[. ..] »^{56}$.

32 Au prix d'adaptations, de changement dans l'équilibre des pouvoirs, de collaboration avec des fonctionnaires venant de l'extérieur (envoyés du gouvernement pendant les périodes de pré-guerre et de guerre, officiers étrangers pendant les deux occupations), les fonctionnaires et administrateurs locaux sont arrivés à diriger le département et à le protéger autant qu'il était possible. Malgré le poids croissant des militaires dans l'administration locale, les civils sont parvenus à conserver une partie de leurs attributions et éviter que le département soit entièrement administré par des 
militaires, et à éviter une vacance du pouvoir. Mais ceci ne s'est fait que grâce à l'augmentation des attributions des administrateurs locaux les plus proches des citoyens, les maires, et grâce à la participation effective des citoyens, et notamment des notables à l'administration locale. Dans certains cas, lors de la seconde occupation notamment, les notables locaux ont même pris directement en main l'administration locale, remplaçant les fonctionnaires dans certains domaines. Cette période de crise confirme donc avec éclat le rôle des notables dans l'administration locale. Administrer un département en temps de crise demande d'avoir un certain relais auprès du peuple. Lorsque les fonctionnaires civils peinent à assumer cette fonction, parce qu'ils sont absents ou parce qu'ils ont perdu la confiance de leurs administrés, le gouvernement ou les habitants se tournent vers les notables locaux, seuls à même, par leur pouvoir, par leur influence locale et par leur ascendant sur leurs concitoyens, de jouer le rôle d'intermédiaires entre l'État et les habitants, rôle d'intermédiaire rendu encore plus nécessaire par les circonstances de crise et l'affaiblissement, ou la désorganisation, de l'État et de ses représentants.

\section{NOTES}

1.Yves-Marie BERCÉ (dir.), La fin de l'Europe napoléonienne, 1814 : la vacance du pouvoir (Actes du colloque de Reims, 28 et 29 septembre 1989), Paris, 1990.

2.Le département commence à se préparer à la guerre dès la fin de 1813, mais la préparation s'accélère après la prise de Genève en janvier 1814. La guerre éclate en Isère en avril 1814, mais s'achève très vite, après que le Sénat ait voté la déchéance de l'Empereur le 12 avril. Commence alors une période d'occupation de deux mois, jusqu'à fin mai 1814, suivie par la première Restauration. L'entrée de Napoléon dans Grenoble, le 7 mars 1815, inaugure les Cent Jours en Isère, qui se concluent, comme l'Empire, par la guerre et l'occupation du département par les troupes autrichiennes et sardes. Ces derniers évacuent le département fin décembre 1815 après environ six mois d'occupation.

3.Jean SAVANT, Les préfets de Napoléon, Paris, 1958, p. 203.

4.Rapport du préfet sur la situation du département au Ministre de l'Intérieur du gouvernement français, 8 mai 1814 (AN, F1cIII/Isère/5).

5.Jean-Luc SuISSA, Le département de l'Eure sous le Consulat et l'Empire (1799-1815), Evreux, 1983, p. 102.

6.François de LANNOY, L'administration préfectorale de la Manche sous le Consulat et l'Empire (1800-1815), Paris, 1992, t. III, p. 658.

7.Nicolas RICHARDSON, The French Prefectoral Corps, 1814-1830, Cambridge, 1966, p. 104.

8.Id., p. 104.

9.Le Ministre de l'Intérieur, Carnot, écrit ainsi aux préfets, le 22 avril 1815 : «Plusieurs individus qui, en d'autres circonstances, pourraient continuer leurs fonctions, ne conviennent pas maintenant, soit pour avoir été compromis depuis un an, soit à raison de leurs faiblesse, soit par la nature de leurs relations sociales [...]» (Lettre du Ministre 
de l'Intérieur aux préfets, 22 avril 1815, AN, F1a31, citée par Nicolas RICHARDSON, op. cit., p. 104).

10.Lettre du directeur des contributions directes au préfet, 2 mai 1815 (ADI, 52M7).

11.Rapport de la commission administrative départementale au Ministre de l'Intérieur, 15医juillet 1815 (ibid., 52M8).

12.Arrêté de la commission administrative départementale, 13 juillet 1815 (ibid., 52M8).

13.Lettre du préfet au Ministre de l'Intérieur, 9 août 1815 (ibid., 181M75).

14.Philippe PIVIDORI, « Les rapports entre les préfets et les chefs militaires en Champagne-Ardenne, 1914-1918 ", dans Maurice VAïssE (dir.), Les préfets, leur rôle, leur action dans le domaine de la défense, de 1800 à nos jours, Paris, 2001, pp. 249-28, p. 250.

15.Lettre du général de brigade Daumas au préfet, 3 janvier 1814 (ADI, 51M16).

16.Catherine LeCOMTE, «Préfets et territoires occupés dans un département stratégique, la Seine-et-Oise ", dans Jean-Pierre AllinNe et Renaud CARRIER (dir.), Préfet et développement local, Paris, 2002, pp. 137-149, p. 140.

17.« Il est urgent qu'il y ait un préfet actif dans le département de l'Isère. Les gardes nationales ne se forment pas. Ordonnez à Bourdon de rester à Grenoble (comme préfet) [...] » (lettre de Napoléon au comte Carnot, Ministre de l'Intérieur, 2 mai 1815. A.N., $\mathrm{F} 1 \mathrm{~b} / 2 /$ Isère/4).

18.Vincent CUVILLIERS, «Les premiers préfets du Pas-de-Calais », dans Annie CRÉPIN, Jean-Marc GuIsuin, Alain LotTIN (dir.), Intendants et préfets dans le Nord Pas de Calais (XVIIeXXe siècle), Arras, 2002, pp.119-135, p. 128.

19.Lettre du commissaire du gouvernement chargé par intérim du portefeuille de l'Intérieur au préfet, 26 juin 1815 (ADI, 52M7).

20.Thierry LeNTZ, Denis IMHOFF, La Moselle et Napoléon, Metz, 1986, p. 259.

21.Lettre du maréchal de camp au maire de Grenoble, 24 juin 1815 (ADI, 52M7).

22.Lettre du commissaire du gouvernement chargé par intérim du portefeuille de l'Intérieur au préfet, 26 juin 1815 (ibid.).

23.Jean CLINQUART, L'administration des douanes en France sous le Consulat et l'Empire (1800-1815), Paris, 1979, pp. 229-230.

24.Lettre du maire de Pont-de-Beauvoisin au préfet, 30 décembre 1813 (ADI, 51M16).

25.Jean CLINQUART, op. cit., pp. 225-227.

26.Lettre anonyme au préfet de l'Isère, janvier-avril 1814 (ADI, 51M5).

27.Lettre du contrôleur principal des Droits Réunis de Vienne au préfet, 14 janvier 1814 (ibid., 51M16).

28.Paul JANSSENS, « Le changement de régime dans les départements belges en 1814 », dans Yves-Marie BERCÉ (dir.), La fin de l'Europe napoléonienne, op. cit., pp. 79-107, p. 80.

29.Paul JANSSENS, op. cit., p. 81.

30.Paul JANSSENS, op. cit., pp. $82-85$ et p. 86.

31.Lettre du directeur des Droits Réunis au préfet, 19 janvier 1814 (ADI, 51M3).

32.Lettre du préfet au Ministre de l'Intérieur, sans date (janvier 1814), (AN, F7/7022).

33.Lettre du préfet au maire de Lumbin, 16 janvier 1814 (ADI, 181M65).

34.Lettre du maire de Pont-de-Beauvoisin au préfet, 5 janvier 1814 (ADI, 51M16).

35.Jacques-Olivier BouDON, «Le corps préfectoral entre deux pouvoirs, 1814-1815 », dans Maurice VAÏsSE (dir.), Les préfets, leur rôle, leur action dans le domaine de la défense ..., op. cit., pp. 13-25 (p. 24).

36.Lettre du maire de Malbenc au préfet, 22 juin 1814 (ADI, 181M68).

37.Lettre de l'adjoint au maire de Voiron au préfet, 5 décembre 1819 (ibid., 51M16). 
38.Lettre du garde général de l'arrondissement de Sassenage au maire de Mens, 12 février 1814 (ibid., 51M21).

39.Jean CLINQUART, op. cit., pp. 231-234.

40.Berriat SAINT PRIX, Napoléon à Grenoble. Histoire du 7 mars 1815, Grenoble, 1861, p. 4.

41.Lettre du contrôleur principal des Droits Réunis de Vienne au préfet, 14 janvier 1814 (ADI, 51M16).

42.Lettre du sous-préfet de Vienne aux maires de l'arrondissement, 19 Janvier 1814 (ibid., 51M3).

43.Lettre du maire de Pont-de-Beauvoisin au préfet, 23 janvier 1814 (ibid., 51M16).

44.Lettre anonyme au préfet, sans date (janvier-avril 1814. ibid., 51M5).

45.Lettre du maire de Pont-de-Beauvoisin au préfet, 23 janvier 1814, op. cit.

46.Jean-François SouLET, Les premiers préfets des Hautes-Pyrénées (1800-1814), Paris, 1965, p. 224.

47.Lettre du maire de Voiron au préfet, 5 avril 1815 (ADI, 52M5).

48. Lettre du préfet au sous-préfet de Saint Marcellin, 16 avril 1815 (ibid., 52M7).

49.Georges CLAUSE, « 1814 : la Champagne entre les armées et les pouvoirs », dans YvesMarie BERCÉ (dir.), La fin de l'Europe napoléonienne, op. cit., pp. 245-274, p. 258.

50.Lettre du chambellan de Sa Majesté Impériale et Royale d'Autriche, à la commission départementale de l'Isère, 11 juillet 1815 (ADI, 52M8).

51.Lettre du président de la commission administrative au vice-intendant, 21 juillet 1815 (ibid., 52M8).

52.Rapport des membres de la commission administrative du département au Ministre de l'Intérieur, 26 juillet 1815 (ibid., 52M8).

53.Rapport des membres de la commission administrative au Ministre de l'Intérieur, 20 juillet 1815 , op. cit.

54.Lettre du maire de Voreppe aux membres du comité de subsistances de Grenoble, 7 août 1815 (ADI, 51M21).

55.Arrêté du conseil municipal de Grenoble, 8 juin 1815 (ibid., 51M21).

56.Lettre du sous-préfet de Vienne au commissaire général de police, 23 février 1816

(ibid., 52M13).

\section{RÉSUMÉS}

Une partie du territoire français, dont l'Isère, est occupée par les Alliés à deux reprises, en 1814 et à nouveau en 1815. La guerre et l'occupation ont des conséquences importantes sur l'administration locale, tant au niveau du personnel et du travail administratifs qu'à celui des relations entre l'État et les citoyens. Ces circonstances de crise sont l'occasion de juger de l'efficacité du modèle administratif français établi par Napoléon le 28 pluviôse an VIII et confronté au risque d'affaiblissement et de désorganisation face à la pression de la guerre et de l'occupation. L'étude de l'administration du département de l'Isère en cette période troublée permet de montrer que la continuité administrative est due pour beaucoup à l'action des administrateurs locaux (surtout les maires) et à la participation des citoyens, notamment des notables. Le modèle administratif qui résiste à cette crise n'est pas celui d'une administration 
jacobine très centralisée, mais bien celui d'une administration véritablement locale, et la résistance du modèle à cette crise montre son efficacité.

Local Government in Time of Crisis: the case of the Isère Department in 1814-1815. Part of French territory, including Isère, was twice occupied by the Allies in 1814 and again in 1815. War and occupation had a major impact on local government, in terms of administrative staff and their work, and the relations between the state and its citizens. These crisis conditions provide an opportunity to assess the efficiency of the French administrative model set up by Napoleon on 28 Pluviôse Year VIII and exposed to the wear and tear of the effects of war and occupation. Study of local government in the Isère department during these troubled times shows that administrative continuity was ensured by the action of local officials (especially the mayors) and the participation of citizens, in particular local worthies. The administrative model that proved resilient in time of crisis was not the highly centralized Jacobin model, but rather that of a truly local administration, a model whose ability to weather the storm is proof of its efficiency.

INDEX

Mots-clés : occupation, guerre, Empire, administration locale, Gouvernance

\section{AUTEUR}

\section{MARIE-CÉCILE THORAL}

Université Pierre Mendès-France

Centre de recherche LAHRHA

Université Pierre Mendès-France, département d'histoire

B.P. 47

38040 - Grenoble Cedex 9 